\title{
Toward Fast Reasoning for Fuzzy Logic Inference
}

\author{
Hung-Pin Chen Jui-Ching Shyur Tai-Ming Parng \\ Department of Electrical Engineering \\ National Taiwan University \\ Taipei, Taiwan, 10764 R.O.C. \\ d79043@cad.ee.ntu.edu.tw
}

\begin{abstract}
This paper presents a method for reducing computations in fuzzy logic inference. Reduction of computations is achieved via three points: (1) avoid match-degree computations over the whole universe, (2) remove redundant match-degree calculation in common if-clauses, and (3) omit calculations of the same fuzzy value in the 'then-part' of different rules. By using these techniques, we show several times improvements over previous methods by Truck Backer Upper Control Systems. We also note that no information of linguistic concept is lost in proposed method.
\end{abstract}

\section{INTRODUCTION}

Recently fuzzy logic has been successfully used in various applications $[2,3]$. This encourages its complex applications that have large amounts of rules and require real-time responses [4]. Since these systems require extensive computations, the computation time becomes a critical issue.

To reduce the number of computations in fuzzy logic inference, a variety of techniques have been proposed $[2,5]$. They are used as follows: (1) In if-part: only singleton signal is permitted and table look-up technique is usually used [1]. (2) In then-part: linguistic value descriptions of then-clauses are replaced with mathematical equations $[2,5]$. Although these methods as above can improve computations in fuzzy logic inference, some linguistic concept informations of fuzzy rules are lost. From these observations, and by focusing on fuzzy logic inference itself, we propose three points that greatly help reducing the number of computations without loss of any information:

(1) Perform computations only on joint parts of non-zero degree input facts and if-clauses.

We record the begin and end indices of non-zero elements of the input facts. Therefore, the begin and end indices of the input fact and each fuzzy value for a variable can be checked to indicate whether there is overlap or not. If overlap exists, its match-degree is evaluated only on joint parts.

(2) Evaluate common if-clauses only once and skip any rule that has a zero match-degree.

Since the same if-clause may appear in more than one rule, its match-degree computation needs to be done only once. The match-degree is broadcasted to the same if-clause in different rules. Meanwhile, we take the minimum of match-degrees as the firing strength of a fuzzy rule. For a given fact, only a few fuzzy values of the input variable may have non-zero match-degrees. Those rules which have if-clauses with zero match-degrees can thus be skipped.

(3) Derive common then-clause in different rules only once.

As the same fuzzy value usually appears in the then-clauses of many rules, if these rules are fired, only the result of the one whose if-part has the maximum firing-strength is effective. Therefore, it is recorded during if-part evaluation from those rules which include the then-clause. As a result, the operation for processing the then-clause with the maximum match-degree is done only once.

For the rest of this paper, the improved fuzzy logic inference is presented in Section 2. In Section 3, the example of fuzzy logic inference is described. Simulation results and comparisons of the Truck Backer-Upper Control System are listed in Section 4. Finally, we make concluding remarks in Section 5. 


\section{IMPROVED METHOD OF FUZZY LOGIC INFERENCE}

In this subsection, we presented our method to fuzzy logic inference. The basic ideas of our method are for efficiently evaluating the match-degrees of if-clauses and the firing strengths of if-parts, and for effectively formulating conclusion as well as fast executing defuzzification. They are discussed in the following subsections.

\subsection{Match-Degree And Firing Strength Evaluation}

The same if-clauses usually appear in the if-parts of different rules, since the fuzzy rules are propositional to fuzzy values of variables. The number of the same if-clauses of if-parts is propositional to the number of variables and its fuzzy values. We assume that the fuzzy values of variable $x_{j}$ are $\left\{\right.$ Fuzzy-Value $_{h}$ of input variable $\left.x_{j}\right\}$, which are denoted by $\left\{F V_{h}\left(x_{j}\right)\right\}, h \in\left\{1, \cdots, l_{j}\right\}, 1 \leq j \leq n$, where $l_{j}$ is the number of fuzzy values of variable $x_{j}$. Then

$$
A_{i j} \in\left\{F V_{h}\left(x_{j}\right)\right\}, 1 \leq i \leq m, 1 \leq j \leq n, h \in\left\{1, \cdots, l_{j}\right\}
$$

where $m$ is the number of fuzzy rules.

When input facts occur, they are not directly broadcasted to if-clauses of their variables. We propose the non-zero range indices for input facts : $B_{x_{j}}$ and $E_{x_{j}}$ to record the begin and end indices of the fact associated with the input variable $x_{j}$. Then boundary checking operations of fact and all fuzzy values of the same variable are computed. Meanwhile, we also propose the non-zero overlapping range indices : $B_{x_{j}}^{\prime}$ s and $E_{x_{j}}^{\prime}$ s to record the non-zero common ranges of the input fact and all fuzzy values of $x_{j}$. Under such circumstance, the universe of discourse is $U_{x_{j}}^{\prime}$ instead of $U_{x_{j}} ; U_{x_{j}}^{\prime}=\left(B_{x_{j}}^{\prime}, \cdots, E_{x_{j}}^{\prime}\right)$.

Accordingly we only calculate the non-zero match-degrees of the input facts and $F V_{h}\left(x_{j}\right)$ s, which are indicated to have overlap during boundary checking operations, we have:

$$
\alpha_{k^{\prime}}\left(x_{j}\right)=\max _{u \in U_{a_{j}}^{\prime}} \min \left(\mu_{F V_{h}\left(x_{j}\right)}(u), \mu_{A_{j}^{\prime}}(u)\right), h \in\left\{1, \cdots, l_{j}\right\}, 1 \leq j \leq n
$$

When these non-zero match-degrees are evaluated, they are broadcasted to some if-clauses which involve the fuzzy value $F V_{h}\left(x_{j}\right)$. So the firing strength of $R_{i}$ can also be derived:

$$
\alpha_{i T}=\min _{j \in\{1, \cdots, n\}}\left(\alpha_{h}\left(x_{j}\right)\right), 1 \leq i \leq m, h \in\left\{1, \cdots, l_{j}\right\}
$$

The computations of formula 2 and 3 can save many fuzzy set operations.

\subsection{Formulating Conclusion}

Since the then-part operations that are just on non-zero elements are effective, the formula is

$$
\mu_{B_{i k}^{\prime}}(v)=\min \left(\alpha_{i T}, \mu_{B_{i k}}(v)\right), v \in V_{y_{k}}^{\prime}
$$

Note that $V_{y_{k}}^{\prime}=(B, E)$ is a subset of $V_{y_{k}}$. For the output variable $y_{k}$, its conclusion is

$$
\mu_{B_{k}^{\prime}}(v)=\bigcup_{i=1}^{m} \mu_{B_{i k}^{\prime}}(v)=\bigcup_{i=1}^{m} \min \left(\alpha_{i T}, \mu_{B_{i k}}(v)\right), v \in V_{y_{k}}^{\prime \prime}, 1 \leq k \leq p
$$

where $V_{y_{k}}^{\prime \prime}=\left(B^{\prime}, E^{\prime}\right)$ is the maximum union subset of the universe for $y_{k}$.

We assume that the fuzzy values of $y_{k}$ are $\left\{\right.$ Fuzzy-Value $_{g}$ of output variable $\left.y_{k}\right\}$, which are denoted by $\left\{F V_{g}\left(y_{k}\right)\right\}, g \in\left\{1, \cdots, q_{k}\right\}$, where $q_{k}$ is the number of fuzzy values of output variable $y_{k}$. Then

$$
B_{i k} \in\left\{F V_{g}\left(y_{k}\right)\right\}, g \in\left\{1, \cdots, q_{k}\right\}, 1 \leq i \leq m, 1 \leq k \leq p .
$$


The fuzzy value $F V_{g}\left(y_{k}\right)$ can appear in the then-clauses of different rules. In other words, some rules may have the same then-clause. When firing strengths of these rules have been evaluated, only the maximum firing strength associated with the fuzzy value $F V_{g}\left(y_{k}\right)$ is effective and necessary to keep during if-part-evaluation. Then the conclusion can be obtained according to the following theorem:

Theorem : The conclusion of the output variable $y_{k}$ is formulated by

$$
\begin{aligned}
\mu_{B_{k}^{\prime}}(v) & = \begin{cases}\bigcup_{g \in\left\{1, \cdots, q_{k}\right\}} \min \left(\alpha_{t_{g} T}, \mu_{F V_{g}\left(y_{k}\right)}(v)\right), v \in V_{y_{k}}^{\prime \prime} & \text { if } \alpha_{t_{g} T}>0 \\
\text { null operation } & \text { otherwise }\end{cases} \\
\alpha_{t_{g} T} & =\max _{i \in T_{g}}\left(\alpha_{i} T\right), 1 \leq i \leq m, g \in\left\{1, \cdots, q_{k}\right\}
\end{aligned}
$$

Proof : Refer to technical report [7].

When the conclusion is obtained, we first calculate the local value of defuzzification $B_{l}^{*}$ only on the subset $\left(B^{\prime}, E^{\prime}\right)$. Then this local value $B_{l}^{*}$ is then added by an amount of $B^{\prime}$ to obtain the defuzzification result.

\section{A SIMPLE EXAMPLE}

In the following, a simple example associated with graph representations is presented to give an intuitive explanation. The fuzzy system has two input variables $x_{1}$ and $x_{2}$ and one output variable $y$. Their fuzzy values are $\left\{A_{1}, A_{2}, A_{3}\right\},\left\{B_{1}, B_{2}, B_{3}\right\}$, and $\left\{C_{1}, C_{2}, C_{3}\right\}$, respectively. There are six fuzzy rules. When facts $A^{\prime}$ of $x_{1}$ and $B^{\prime}$ of $x_{2}$ are given, the fuzzy logic rules are inferred. The original method and our method are shown in Figure 1 and 2, respectively. When fuzzy rules have been inferred, the conclusion $C^{*}$ of $y$ is obtained. Meanwhile, the equivalence of our method and the original method is shown. In our method, the linguistic information of fuzzy rules are still kept, and the computations are effectively reduced.

\section{AN APPLICATION}

The experiment has been conducted for the Truck Backer-Upper control systems. which is presented by [6], has two input state variables and one output control variable, with 35 rules. The three state variables $\phi, X$, and $Y$ determine the truck position with $\phi$ specifying the angle of the truck with the horizontal. The coordinate pair $(x, y)$ specifies the position of the rear center of the truck. The control variable was the steering-angle signal, $\theta$. The coordinate $X$ and $Y$ range from 0 to $1000, \phi$ ranges from -90 to 270 , and $\theta$ ranges from -30 to 30 . Positive values of $\theta$ represent clockwise rotations of the steering wheel. The resolution of $\phi$ and $\theta$ was $1^{\circ}$ each. The resolution of $X$ and $Y$ was 1 . And $r$ is set to 20. The fuzzy values of membership function of $\phi, X$, and $\theta$ variables are the same as those given in [6], which are shown in Figure 3. The FAM-bank rule description of the control system (refer to [6]) is shown in Figure 4, e.g. Rule 1 : if $\phi$ is RB and $X$ is LE then $\theta$ is PS. For an input fact, a crisp value from a real world device can usually be inferred to be a fuzzy value by taking into account the typical error information that is specified by device's manufacturer. So, the input facts of $\phi, X$, and $Y$ are crisp values fuzzified with base widths 5,7 , and 7 , respectively.

Three cases are tried in each experiment. The initial positions $(x, y, \phi)$ from case 1 to case 3 are $(200,200,30),(300,100,220)$, and $(300,400,-10)$. The destination position $(x, y)$ is $(500,1000)$. All simulated trajectories are shown in Figure 5. The results of fuzzy logic inference based on our approach, compared with the originai method, are shown in Table I. Note that the (A) term, (B) term, and (C) term in the original method mean the number of match-degree evaluations, firing strength evaluations, and active rules, respectively. Meanwhile, the (D) term, (E) term, (F) term, and (G) term in our method mean the number of fuzzy value evaluations, firing strength evaluations, active rules, and fuzzy value derivations multiplied with its fuzzy subset rate, respectively. 


\section{CONCLUSION}

In this paper, we have presented our method toward fast fuzzy logic inferences. Fuzzy set operations are only in fuzzy value evaluation and then-clause derivation. Only one fuzzy set operation is necessary for the fuzzy value overlapped with the input fact and for the fuzzy value associated with non-zero firing strength. The type of if-part evaluations of fuzzy rules is converted from fuzzy set operations to real value operations. By using these techniques, we show several times improvements over previous methods by application systems. We also note that no information of linguistic concept is lost in proposed method.

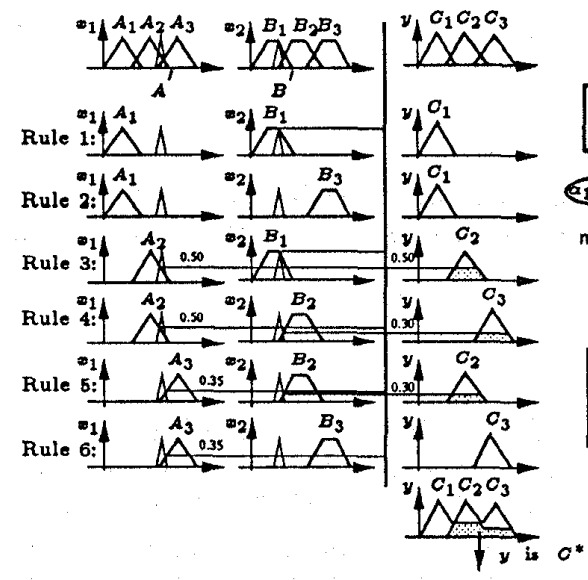

Figure 1. The original method.
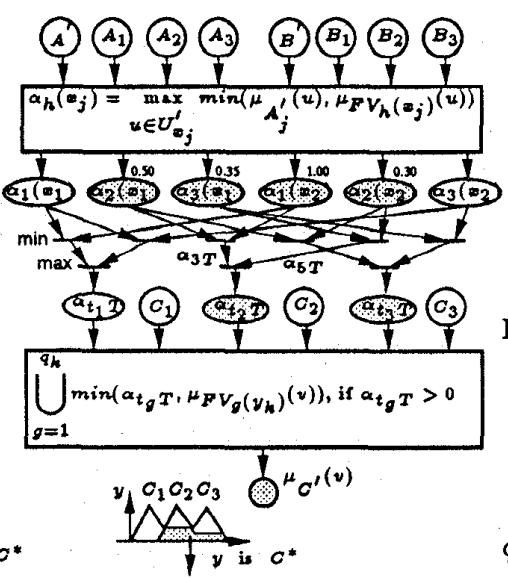

Figure 2. Our method.

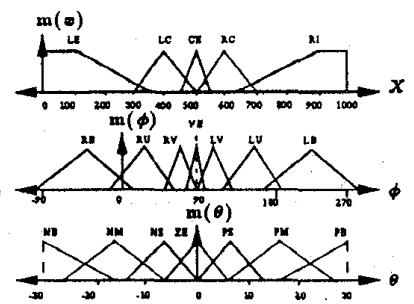

Figure 3. Membership graphs.

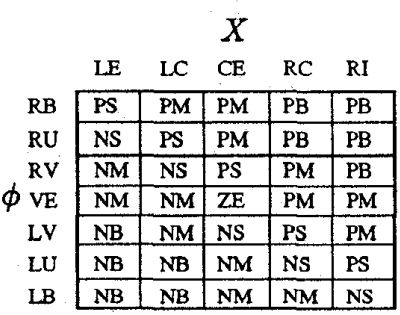

Figure 4. FAM-bank matrix.

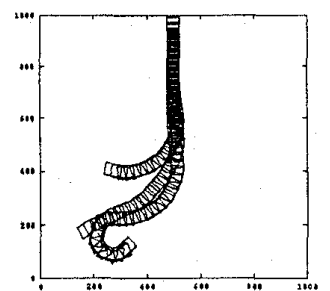

Figure 5. Truck trajectories.

\section{References}

[1] Tri-cker Chiueh, "Optimization of Fuzzy Logic Inference Architecture," IEEE Computer Magazine, May 1992.

[2] Lee, C. C., "Fuzzy Logic in Control Systems," IEEE Trans. on Systems, Man and Cybernetics, Vol. 20, No. 2, 1990.

[3] C. Bellon, P. Bosc, and H. Prade, "Fuzzy Boom in Japan," International Journal of Intelligent Systems, Vol. 7, 1992, pp. 293-361.

[4] C. von Altrock, B. Krause and H.-J, Zimmermann, "Advanced fuzzy logic control of a model car in extreme situations," Fuzzy Sets and Systems, Vol. 48, 1992, pp. 41-52.

[5] J.-S.R. Jang and C.-T. Sun, "Functional Equivalence Between Radial Basis Function Networks and Fuzzy Inference Systems," IEEE Trans. on Neural Networks, Vol. 4, No. 1, January, 1993, pp. 156-159.

[6] S.-G. Kong and B. Kosko, "Adaptive Fuzzy Systems for Backing up a Truck-and-Trailer," IEEE Trans. on Neural Networks, Vol. 3, No. 2, Mar. 1992, pp. 211-223.

[7] Hung-pin Chen, "Technical report," Aug. 1992. 